\title{
ARBITRARILY ACCURATE APPROXIMATE INERTIAL MANIFOLDS OF FIXED DIMENSION
}

\author{
James C. Robinson \\ Department of Applied Mathematics and Theoretical Physics, \\ Silver Street, Cambridge, CB3 9EW, \\ England.
}

\begin{abstract}
By employing an embedding result due to Mañé, and its recent strengthening due to Foias \& Olson it is shown that a global attractor with finite fractal (box counting) dimension $d$ lies within an arbitrarily small neighbourhood of a smooth graph over the space spanned by the first $[[2 d+1]]$ Fourier-Galerkin modes. The proof is, however, non-constructive.
\end{abstract}




\section{Introduction}

For many dissipative partial differential equations, the long term dynamics is determined by the behaviour on a much smaller set, the global attractor $\mathcal{A}$. This is the maximal compact invariant set that attracts all bounded sets, see Refs. [1] and [2] for many examples. In many cases it can also be shown that the attractor has finite fractal (box counting) dimension [3]. Such systems are commonly analysed in the form of an abstract evolution equation on a Hilbert space $H$,

$$
d u / d t+A u=f(u)
$$

where $A$ is a positive self-adjoint operator with compact inverse and $f$ a nonlinear term with some Lipschitz smoothness properties. This general semilinear parabolic equation covers many examples and forms the main subject of the monograph [4]. In what follows, the norm in $H$ will be denoted by $|\cdot|$ and the inner product by $(\cdot, \cdot) . H$ has a natural basis consisting of Fourier modes, the eigenfunctions $w_{n}$ of the operator $A\left(A w_{n}=\lambda_{n} w_{n}\right)$. By ordering the eigenvalues in increasing order $\left(\lambda_{n+1} \geq \lambda_{n}\right)$, one can define the projection $P_{n}$ onto the first $n$ Fourier modes,

$$
P_{n} u=\sum_{j=1}^{n}\left(u, w_{j}\right) w_{j}
$$

and its orthogonal complement $Q_{n}=I-P_{n}$.

For some systems one can show that the global attractor is contained in a manifold, given as the graph of a $C^{1}$ function over the finite-dimensional space $P_{n} H$, which is positively invariant under the flow and attracts all the orbits exponentially. This is termed an inertial manifold [5], and has been found to exist for many equations. Nevertheless, the spectral gap condition (a sufficient condition for existence, but one required by all current proofs for equations of a general form, see for example [6]) prevents this result being verified for some important examples, including the two-dimensional Navier-Stokes equations. Even when an inertial manifold exists, the abstract existence results do not give an explicit form for the manifold, showing only that there exists a function $\phi: P_{N} H \rightarrow Q_{N} H$ whose graph

$$
\left\{p+\phi(p): p \in P_{N} H\right\}
$$


is an inertial manifold. To remedy this, the idea of an "approximate inertial manifold" was introduced in Refs. [7] \& [8]. In this case, the global attractor is shown to lie in some small neighbourhood of an explicitly given manifold, the width of this neighbourhood shrinking, preferably rapidly, as the dimension of the manifold increases.

In Ref. [7] a non-constructive proof was given that there exists a family of approximate inertial manifolds $\mathcal{M}_{N}$ of exponential order, i.e. such that

$$
\operatorname{dist}\left(\mathcal{M}_{N}, \mathcal{A}\right) \leq C e^{-k \lambda_{N}^{\alpha}}
$$

where $\mathcal{M}_{N}$ is an $N$-dimensional manifold (and $C, k, \alpha$ are all positive constants). Each manifold is the graph of a Lipschitz function $\phi_{N}: P_{N} H \rightarrow$ $Q_{N} H$ with Lipschitz constant at most 1. (An explicit scheme for constructing such a family with exponential order was given later in Ref. [9].)

In this paper it is shown that there exist arbitrarily accurate approximate inertial manifolds at any fixed dimension, provided the dimension is higher than (approximately) twice the dimension of the global attractor. To compensate for this improvement, an increasingly accurate sequence of manifolds $\mathcal{M}_{\epsilon}$, with

$$
\operatorname{dist}\left(\mathcal{M}_{\epsilon}, \mathcal{A}\right) \leq \epsilon
$$

has diverging Lipschitz constant as $\epsilon \rightarrow 0$.

This result, independent of any conditions, explains two potentially puzzling results that arise in the theory of approximate inertial manifolds (see, for example, Ref. [10]). In systems for which no true inertial manifold can be shown to exist, it is often possible to show the existence of an approximate inertial manifold; and for systems which do have an exact inertial manifold, approximate inertial manifolds generally have much smaller dimension.

\section{Embedding finite-dimensional sets in $\mathbb{R}^{D}$}

Although the global attractor is finite dimensional, it lies within the infinite-dimensional Hilbert space $H$. However, in [3] Mañé showed that "most" projections of a set $X$ with a finite fractal dimension $d_{F}(X)=d$ onto subspaces of dimension $[[2 d+1]]$ are injective $([[k]]$ denotes the smallest integer greater than or equal to $k$ ). The best version of the theorem is due to Foias \& Olson [11], who prove Hölder continuity for the inverse of the projection. 
Theorem 1 (Mañé, Foias \& Olson). If $H$ is a Hilbert space and $X$ is a compact subset of $H$ with $d_{F}(X) \leq d$ then given an orthogonal projection $P_{0}$ of rank $[[2 d+1]]$, for every $\delta>0$ there exists an orthogonal projection $P=P(\delta)$ of the same rank such that

$$
\left\|P-P_{0}\right\| \leq \delta
$$

and $P$ is injective on $X$. Furthermore there exist constants (depending on б) $C>0$ and $0<\theta<1$ such that

$$
\left|P^{-1} x-P^{-1} y\right| \leq C|x-y|^{\theta} \quad x, y \in P X .
$$

It is of course immediate from this theorem that one can write the attractor as a graph over a subspace of $P H(P \mathcal{A}$, in fact), so that

$$
X=\{\pi+\phi(\pi): \pi \in P \mathcal{A}\}
$$

where $\phi(\pi)=\left(P^{-1} \pi\right)-\pi$ is Hölder continuous for $\pi \in P \mathcal{A}$. If one extends $\phi$ to a function defined over the whole of $P H$, using the extension theorem in [12] which guarantees that the extension is also Hölder and bounded by its maximum value on $P \mathcal{A}$, it is clear that the global attractor $\mathcal{A}$ lies within a Hölder continuous manifold,

$$
X \subset \mathcal{G}_{\phi} \equiv\{\pi+\phi(\pi): \pi \in P H\}
$$

This is reminiscent of the results of Refs. [13] and [14], which show that the attractor for a reaction-diffusion equation is a smooth graph. Note, however, that the graph of $\phi$ is not (Lipschitz) smooth nor is it positively invariant under the dynamics, unlike an inertial manifold.

\section{Approximate inertial manifolds}

Direct application of theorem 1 to the global attractor leads to (1). Indeed, theorem 1 implies that such a statement is possible with $P$ arbitrarily close to the distinguished projection $P_{N}$. However, in every case the particular projection $P$ is unknown.

Turning this around, one can show that (1) holds "arbitrarily accurately" when $P$ is replaced by the Fourier projection $P_{N}$. This observation 
relates the embedding result of Foias \& Olson to the theory of approximate inertial manifolds.

It will be shown that there exists a function $\psi: P_{N} H \rightarrow Q_{N} H$, such that the global attractor lies within an arbitrarily small neighbourhood of the graph of $\psi$,

$$
\mathcal{G}_{\psi}=\left\{p+\psi(p): p \in P_{N} H\right\}
$$

Theorem 2. Let $\mathcal{A}$ be a compact set with $d_{F}(\mathcal{A}) \leq d$, and $N \geq[[2 d+1]]$. Then given $\epsilon>0$ there exists a Lipschitz function $\psi: P_{N} H \rightarrow Q_{N} H$, with graph $\mathcal{G}_{\psi}$ as above, such that

$$
\operatorname{dist}\left(\mathcal{A}, \mathcal{G}_{\psi}\right) \leq \epsilon
$$

Proof. Since $\mathcal{A}$ is compact it is bounded, and so $\mathcal{A} \subset \Omega_{\rho} \equiv\{|u| \leq \rho\}$ (which implies that $\left.\|\phi\|_{\infty} \leq \rho\right)$. Now, given an $\epsilon>0$, use theorem 1 to choose a projection $P$ which is injective on $\mathcal{A}$ and which satisfies

$$
\left\|P-P_{N}\right\| \leq \epsilon / 6 \rho \text {. }
$$

Note that it follows that $\left\|Q-Q_{N}\right\| \leq \epsilon / 6 \rho$ also. Given such a $P$, there is a corresponding $\phi: P H \rightarrow Q H$ as in (1), such that $\mathcal{A} \subset \mathcal{G}_{\phi}$. The aim is now to construct a function $\psi: P_{N} H \rightarrow Q_{N} H$ whose graph is close to $\mathcal{G}_{\phi}$. Now, $P: P_{N} H \rightarrow P H$ is injective, for otherwise there exist $p, \bar{p} \in P_{N} H$ with $p \neq \bar{p}$ and $P(p-\bar{p})=0$, i.e. a $w \in P_{N} H$ with $w \neq 0$ and $P w=0$. But then

$$
\left|P_{N} w-P w\right|=\left|P_{N} w\right|=|w|
$$

contradicting (2). $P$ also maps $P_{N} H$ onto $P H$, since $\left\{w_{j}\right\}_{j=1}^{N}$, the basis for $P_{N} H$, is mapped into $\left\{P w_{j}\right\}_{j=1}^{N}$; and if

$$
\sum_{j=1}^{N} \lambda_{j} P w_{j}=0 .
$$

then the linearity of $P$ and its injectivity on $P_{N} H$ imply that $\lambda_{j}=0$ for all $j$; and hence the $\left\{P w_{j}\right\}$ are linearly independent. They thus form a basis for $P H$, and so $P$ is a bijection from $P_{N} H$ onto $P H$. Similarly, $P_{N}$ maps $P H$ bijectively onto $P_{N} H$. Since $P: P_{N} H \rightarrow P H$ is onto, the set

$$
\left\{P p+\phi(P p): p \in P_{N} H\right\} \equiv\left\{p+[P p-p+\phi(P p)]: p \in P_{N} H\right\}
$$


is exactly the set $\mathcal{G}_{\phi}$ defined above (1). For an approximate inertial manifold in the standard form, take

$$
\psi(p)=Q_{N}(P p-p+\phi(P p))=Q_{N}[P p+\phi(P p)], \quad p \in P_{N} H
$$

and observe that $\psi$ is also Hölder continuous,

$$
\begin{aligned}
|\psi(p)-\psi(\bar{p})| & \leq|P p+\phi(P p)-P \bar{p}-\phi(P \bar{p})| \\
& \leq|p-\bar{p}|+C|p-\bar{p}|^{\theta} .
\end{aligned}
$$

Now, for $p \in P_{N} H$,

$$
\begin{aligned}
|P p+\phi(P p)-p-\psi(p)| & \leq|P p-p|+\left|Q_{N}(p-P p)\right|+\left|\phi(P p)-Q_{N} \phi(P p)\right| \\
& \leq \epsilon(|p|+|p|+|\phi(P p)|) \leq \epsilon / 2
\end{aligned}
$$

for $u \in \Omega_{\rho} \supset \mathcal{A}$. It follows from the bijective properties of $P$ and $P_{N} H$ that the Hausdorff distance between the two sets $\mathcal{G}_{\phi} \cap \Omega_{\rho}$ and $\mathcal{G}_{\psi} \cap \Omega_{\rho}$ is bounded by $\epsilon / 2$, and hence

$$
\operatorname{dist}\left(\mathcal{A}, \mathcal{G}_{\psi}\right) \leq \epsilon / 2 \text {. }
$$

Thus $\mathcal{A}$ lies within an $\epsilon / 2$ neighbourhood of a graph over $P_{N} H$, for arbitrarily small $\epsilon$. The function $\psi$ can be smoothed to produce a Lipschitz (rather than only continuous) manifold - if $\psi_{\epsilon}$ is a smoothing that lies within $\epsilon / 2$ of $\psi$ then clearly

$$
\operatorname{dist}\left(\mathcal{A}, \mathcal{G}\left[\psi_{\epsilon}\right]\right) \leq \epsilon
$$

and this an approximate inertial manifold of the required form.

Note that if the global attractor is known to be a bounded subset of $D\left(A^{r}\right)$, then one can simply replace $H$ in the above argument with $D\left(A^{r}\right)$ and obtain a Lipschitz function into $Q_{N} H \cap D\left(A^{r}\right)$.

\section{Conclusion}

In general, the results of Mañé and Foias \& Olson assert that a finitedimensional global attractor will lie within the graph of a Hölder function over some finite-dimensional space. If one selects the distinguished space $P_{N} H$ spanned by the first $N$ Fourier modes, the global attractor lies within an arbitrarily small neighbourhood of a Lipschitz graph over $P_{N} H$, where $N$ is comparable to the dimension of the attractor. As discussed in the 
introduction, this explains two phenomena from the theory of approximate inertial manifolds.

\section{Acknowledgments}

Many thanks to Trinity College, Cambridge, for their continued financial support and generosity.

\section{References}

[1] J.K. Hale, Asymptotic behaviour of dissipative systems, in: Math. Surveys and Monographs, Vol. 25, Amer. Math. Soc. (Providence, 1988).

[2] R. Temam, Infinite dimensional dynamical systems in mechanics and physics, AMS 68, Springer (London, 1988).

[3] R. Mañé, Springer Lecture Notes in Maths. 898 (1981) 230.

[4] D. Henry, Geometric theory of semilinear parabolic equations, in: Lecture Notes in Math., Vol 840. (Springer-Verlag, New York, 1981).

[5] C. Foias, G.R. Sell and R. Temam, C. R. Acad. Sci. I 301 (1985) 139.

[6] S.-N. Chow, K. Lu and G.R. Sell, J. Math. Anal. Appl. 169 (1992) 283.

[7] C. Foias, O. Manley, and R. Temam, Math. Mod. Num. Anal. 22 (1988) 93.

[8] C. Foias, G.R. Sell, and E.S. Titi, J. Dyn. Diff. Eq. 1 (1989) 199.

[9] A. Debussche and R. Temam, J. Math. Pures Appl. 73 (1994) 489.

[10] M. Marion, J. Dyn. Diff. Eq. 1 (1989) 245.

[11] C. Foias and E. Olson, Indiana Univ. Math. J. 45 (1996) 603.

[12] E.M. Stein, Singular integrals and differentiability properties of functions, Princeton University Press (Princeton, 1970).

[13] M.S. Jolly, J. Diff. Eq. 78, 220.

[14] P. Brunovský, J. Dyn. Diff. Eq. 2 (1990) 293. 